\title{
RATE OF GROWTH OF HURWITZ ENTIRE FUNCTIONS AND INTEGER VALUED ENTIRE FUNCTIONS
}

\author{
BY DAIHACHIRO SATO AND ERNST G. STRAUS
}

Communicated by R. P. Boas, October 28, 1963.

1. Introduction. A Hurwitz function is a function $f(z)$ with $f^{(n)}(0)$ $=$ integer for $n=0,1,2, \ldots$. An integer valued function is a function $g(z)$ with $g(n)=$ integer for $n=0,1,2, \cdots$.

It is well known that every transcendental Hurwitz entire function and every transcendental integer valued entire function must be at least of exponential order, type 1 and $\log 2$ respectively, which are the best possible values. (Example: $f(z)=e^{z}, g(z)=2^{z}$.)

Various improvements on these facts have been studied to a considerable extent. It is the purpose of this note to establish the precise dividing line for the growth of these entire functions below which one finds only polynomials.

2. Hurwitz entire function and integer valued entire function.

Definition. Let

$$
\begin{aligned}
\phi(r) & =\max _{n} \frac{r^{n}}{\Gamma(n+1)} \quad(r \geqq 0) \\
& =\exp \left(r-(\log r) / 2-(\log 2 \pi) / 2+1 /(24 r)+O\left(1 / r^{2}\right)\right) .
\end{aligned}
$$

THEOREM 1. Let $\psi(r)$ be any increasing function such that for every $N$, there exists an $r_{N}$, so that $\psi(r)>r^{N}$ for all $r>r_{N}$, then there exists a nondenumerable set of transcendental Hurwitz entire functions which satisfy

$$
M(r)<\phi(r)+\psi(r)
$$

for all $r>R$, where $R$ is a suitable positive number depending only on $\psi(r)$. Here $M(r)$ is the maximum modulus of $f(z)$ at $|z|=r$.

THeorem 2. A Hurwitz entire function is a polynomial if

$$
M(r)<\phi(r)+r^{N}
$$

for some $N$ and all $r>r_{0}$.

THEOREM 3. There exists a denumerable infinite set of transcendental integer valued entire functions which satisfy

$$
M(r)<2^{r}-r^{N}
$$

for any fixed $N$ and all $r>r_{0}$. 
THEOREM 4. With any $\psi(r)$ as in Theorem 1 , an integer valued entire function is a polynomial if

$$
M(r)<2^{r}-\psi(r)
$$

for all $r>R$.

CoRollary 1. For every $\epsilon>0$ there exists a transcendental Hurwitz entire function with

$$
\limsup _{r \rightarrow \infty} \frac{\sqrt{2 \pi r} e^{-r} M(r)}{\left(1+\frac{1+\epsilon}{24 r}\right)}<1
$$

while every Hurwitz entire function for which

$$
\limsup _{r \rightarrow \infty} \frac{\sqrt{2 \pi r} e^{-r} M(r)}{\left(1+\frac{1-\epsilon}{24 r}\right)} \leqq 1
$$

is a polynomial.

3. Two points Hurwitz entire function. Both authors have considered entire functions all of whose higher derivatives are integral valued at several integral points. While the results are satisfactory as far as the minimal order of such transcendental functions is concerned, we have been able to determine so far the minimal type only in the case of two points. In the particular case in which the functions are Hurwitz entire functions at two consecutive integers, say $z=0$ and $z=1$ (i.e., entire function $f(z)$ for which $f^{(n)}(z)=$ integer at $z=0,1$; $n=0,1,2, \cdots)$, much of the analysis to prove Theorems 1 and 2 can be carried out to give sharper results on their rate of growth.

THEOREM 5. Let $\phi(r)$ and $\psi(r)$ be as in Theorem 1. Then there exists a nondenumerable set of transcendental Hurwitz entire functions at 0 and 1 for which $M(r)<\phi(r(r+1))+\psi(r)$ for $r>R$ where $R$ depends only on $\psi(r)$.

THEOREM 6. If $f(z)$ is a Hurwitz entire function at 0 and 1 which satisfies $M(r) \leqq \phi(r(r-1))$ for all $r>r_{0}$ then $f(z)$ is a polynomial.

CoROLlaRy 2. There exist transcendental Hurwitz entire functions at 0 and 1 with $M(r)<\exp \left(r^{2}+r-\log r+o(\log r)\right)$ while every Hurwitz entire function at 0 and 1 for which $M(r)<\exp \left(r^{2}-r-\log r+o(\log r)\right)$ is a polynomial. 


\section{Indication of proof.}

Proof of Theorem 1. Let $0 \leqq n_{1}<n_{2}<\cdots<n_{i}<\cdots$ be a sequence of integers such that $n_{i+1}>4 n_{i}$ and $\psi(r)>2^{n_{i}-1}$ for any $r>n_{i} / 4$. Then the function $f(z)=\sum_{i=0}^{\infty} z^{n_{i}} / n_{i}$ ! has the described property. It is clear that any infinite subsequence of our sequence $\left\{n_{i}\right\}$ gives rise to a transcendental Hurwitz entire function whose rate of growth is not greater than that of $f(z)$ and there are nondenumerably many such subsequences.

Proof of Theorem 2. For a Hurwitz entire function $f(z)$ $=\sum_{k=0}^{\infty} a_{k} z^{k} / k$ ! we have $a_{k}=$ integer and

$$
(M(r))^{2} \geqq \frac{1}{2 \pi r} \oint_{|z|=r}|f(z)|^{2} \cdot|d z|=\sum_{k=0}^{\infty} \frac{\left|a_{k}\right|^{2}}{(k !)^{2}} r^{2 k} .
$$

Pick $r_{1} \geqq r_{0}$ so that $\phi(r)>r^{N}$ for all $r>r_{1}$ then for any $k>r_{1}$ we have $\left|a_{k}\right| \leqq 1$, since otherwise (i.e., $\left|a_{k}\right| \geqq 2$ ) we should have $(M(r))^{2}$ $=4(\phi(r))^{2}>\left(\phi(r)+r^{N}\right)^{2}$ when $r^{k} / \Gamma(k+1)=\phi(r)$ or $r \approx k+\frac{1}{2}>r_{1}$ contrary to (3). Assume now $\left|a_{k}\right|=1$ for some sufficiently large $k$, then considering $r$ so that $\phi(r)=r^{k} / \Gamma(k+1)$ we have, by (8), that $a_{m}=0$ for all $k / 4<m<k$ wherever $a_{k} \neq 0, k>k_{0}$. If $f(z)$ is transcendental we can pick $k_{0}<k_{1}<k_{2}$ so that $a_{k_{1}} \neq 0, a_{k_{2}} \neq 0$ and $a_{m}=0$ for $k_{1}<m<k_{2}$. This gives that for sufficiently large choices of $k_{1}, k_{2}$ we get $M(r)>\phi(r)$ $+r^{N}$, contrary to hypothesis.

Proof of Theorem 3. For any integer valued polynomial $P(z)$ of degree $n>N$ with a positive leading coefficient, an entire function $g(z)=2^{z}-P(z)$ is a transcendental integer valued entire function which satisfies (4) and we have denumerably infinite set of such functions.

Proof of Theorem 4. The function $g(z)$ must have the form $g(z)$ $=P(z)+Q(z) \cdot 2^{z}$ where $P(z), Q(z)$ are polynomials. The condition (5) requires that $Q(z)$ must be a constant and $|Q| \leqq 1$. The fact that $g(n)=$ integer for $n=0,1,2, \cdots$ gives that $Q$ be a rational number $Q=t / s,(t, s)=1,|t| \leqq s, t, s=$ integer. Considering the periodicity $(\bmod p)$ of $g(z)$, we have that no prime $p$ can be a factor of $s$ and hence $s=1$. This gives that the only possible values of $Q$ be 0 and \pm 1 . If $Q=0$, then $g(z)$ is a polynomial, if $Q= \pm 1$, then $g(z)$ does not satisfy (5).

Proof OF Corollary 1. It is a direct application of the asymptotic expansion (1) with Theorems 1 and 2.

Proof of Theorem 5. If $f(z)$ is a Hurwitz entire function with $M(r)=\chi(r)$, then $g(z)=f(z(z-1))$ is a Hurwitz entire function at 0 and 1 with $M(r) \leqq \chi(r(r+1))$. 
Proof of Theorem 6. Denoting $[t]$ for the greatest integer not exceeding $t$, we can expand $f(z)$ in a series

$$
f(z)=\sum_{n=0}^{\infty} a_{n} z^{[(n+1) / 2]} \cdot(z-1)^{[n / 2]} /[n / 2] !
$$

with the coefficients

$$
a_{n}=\frac{\lfloor n / 2\rfloor !}{2 \pi i} \oint_{|\zeta|=r} \frac{g(\zeta) d \zeta}{\zeta^{[(n+2) / 2]} \cdot(\zeta-1)^{[(n+1) / 2]}} .
$$

Now $f(z)$ is a Hurwitz function at 0 and 1 if and only if $a_{n}=$ integer for $n=0,1,2, \cdots$. If we choose $r$ so that $\phi(r(r-1))$ $=(r(r-1))^{[n / 2]} /[n / 2] !$, then we have

$$
\left|a_{n}\right|<M(r) \cdot[n / 2] ! /\left(r^{[n / 2]}(r-1)^{[(n+1) / 2]}\right) \leqq M(r) / \phi(r(r-1)) .
$$

Thus, if $M(r) \leqq \phi(r(r-1))$ for $r>r_{0}$ then $\left|a_{n}\right|<1$ and hence $a_{n}=0$ for $n>n_{0}$. This means that $f(z)$ is a polynomial.

Proof of Corollary 2. Consequence of Theorems 5 and 6 with asymptotic expansion (1).

\section{Remarks.}

1. While Theorems 1 and 2 give the exact dividing line between the denumerable and nondenumerable sets of Hurwitz entire functions, the similar dividing line for the integer valued entire functions is still unsettled.

2. In view of Corollary 2, we see that there remains the question of the best constant $-1 \leqq c<1$ so that all transcendental Hurwitz entire functions at 0 and 1 must satisfy $M(r)>\exp \left(r^{2}+c r+o(r)\right)$ for arbitrarily large $r$.

3 . We can recapture the precision attained in the one point case if we modify the region in which we maximize $|f(z)|$, by replacing the circle $|z|=r$ by a lemniscate $|z(z-1)|=r^{2}$. Thus we have

TheOREM 7. Let

$$
M^{*}(r)=\max _{|z(z-1)|=r^{2}}|f(z)| .
$$

If $f(z)$ is a Hurwitz entire function at 0 and 1 and $M^{*}(r)<\phi\left(r^{2}\right)+r^{N}$ for some $N$ and all $r>R$, then $f(z)$ is a polynomial. On the other hand, if $\psi(r)$ is as in Theorem 1, then there exists a nondenumerable set of transcendental Hurwitz entire functions at 0 and 1 with $M^{*}(r)<\phi\left(r^{2}\right)+\psi(r)$, $r>R$. 


\section{REFERENCES}

1. L. Bieberbach, Über einen Satz Pólyascher Art, Arch. Math. 4 (1953), 23-27.

2. R. P. Boas, Jr., Entire functions, Academic Press, New York, 1954. ${ }^{1}$

3. R. C. Buck, Integral valued entire functions, Duke Math. J. 15 (1948), 879-891. ${ }^{1}$

4. A. H. Cayford, $A$ class of integer valued entire functions, Dissertation, Univ. of California, Los Angeles, Calif., 1961.

5. S. Kakeya, Notes on the maximum modulus of a function, Tôhoku Math. J. 10 (1916), 68-70.

6. G. Pólya, Über die kleinsten ganzen Funktionen deren sämtliche Derivierte im Punkte $z=0$ ganzzahlig sind, Tôhoku Math. J. 19 (1921), 65-68.

7. D. Sato, Integer valued entire functions, Dissertation, Univ. of California, Los Angeles, Calif., 1961.

8. E. G. Straus, On entire functions with algebraic derivatives at certain algebraic points, Ann. of Math. (2) 52 (1950), 188-198.

9. - Some topics in integer valued functions, Report of the institute of number theory, Boulder, Colorado (1960), pp. 99-103.

University of California, Los Angeles and University of Saskatchewan, Regina Campus

${ }^{1}$ See other references of integer valued entire functions in $[2 ; 3]$ above. 